\title{
Origine e diffusione di caramba e di sbirro
}

\author{
Manuel Favaro
}

PUBBLICATO: 21 SETTEMBRE 2021

\section{Quesito:}

Alcuni lettori ci chiedono notizie sulla diffusione delle parole caramba e sbirro; in merito a sbirro ci è stato anche domandato se vi siano delle differenze tra l'uso che se ne fa oggi rispetto a quello dei secoli passati.

\section{Origine e diffusione di caramba e di sbirro}

\section{Caramba}

ome avverte Ernesto Ferrero nel Dizionario storico dei gerghi italiani: dal Quattrocento a oggi (Milano, Mondadori, I99I), caramba è "una delle tante deformazioni ironiche" della parola carabiniere. Tra gli altri gergalismi usati per riferirsi ai membri dell'Arma lo studioso cita come "egualmente diffuse" carabba, carubba, carrubbi, carrubi. Anche Augusta Forconi, in La mala lingua. Dizionario dello «slang» italiano (Milano, SugarCo, 1988), menziona carabba oltre a caramba, rimandando anche a carubba; anche queste forme vengono segnalate dall'autrice come deformazioni dal "tono scherzoso o spregiativo" di carabiniere.

Molto probabilmente, caramba si è imposto nell'uso rispetto alle altre varianti per via dell'analogia con l'esclamazione caramba!, voce eufemistica di origine spagnola (GDLI, Supplemento 2004; v. anche il DLE, Diccionario de la lengua española, disponibile sul sito dle.rae.es). Con questa accezione il vocabolo si attesta già a partire dal diciannovesimo secolo nell'italiano letterario (due occorrenze in LIZ 200I, appartenenti entrambi al romanzo La bocca del lupo di Remigio Zena, composto alla fine dell'Ottocento), mentre la voce di provenienza gergale fatica a penetrare nella lingua di tutti i giorni.

Per quanto riguarda l'uso giornalistico, la prima testimonianza rinvenuta negli archivi risale a un articolo della "Stampa" del ig69 che tratta della pubblicazione, da parte della Polizia di stato, di un dizionario sul gergo dei criminali da utilizzare come sussidio informativo per la formazione delle reclute; oltre a caramba, che secondo quanto riportato nell'articolo sarebbe stata la forma allora circolante negli ambienti malavitosi di Trento, l'articolo menziona alcune altre possibili varianti con cui i carabinieri venivano appellati dai criminali:

\footnotetext{
A Bari i carabinieri in pattuglia sono chiamati "fratelli Bandiera", mentre da Palermo a Torino gli stessi carabinieri diventano "fratelli Branca". Il carabiniere è "Gianni" a Cagliari, "caraba" a Firenze, [...] "ciapaciuc" ad Aosta, "giusta" a Potenza, "scime" a Bari, "asso di danaro" a Milano, "chiodi" a Roma. Il carabiniere in alta uniforme è a Roma, il "pinguino", con riferimento alla giacca con le code. ([s.f.], Come parla la malavita, "La Stampa", 9/5/1969)
}

Insomma, sul finire degli anni Sessanta la situazione era tutt'altro che unitaria. Gran parte delle successive testimonianze rinvenute nell'archivio sono inserite tra virgolette, all'interno delle battute dialogiche riportate dal giornalista:

Ad un tratto, accanto al marciapiede, si fermavano una "Renault" - targata pare SV I35246 - ed un pulmino "Volkswagen". Sui due veicoli c'era solo il conducente. L'autista della vettura si è sporto dal 
finestrino ed ha apostrofato l'ex appuntato: "Tu sei un caramba, vieni con me". ([s.f.], È rapito in auto nella notte da due uomini ad Albissola, "La Stampa", 7/2/1973)

Oppure, in alcuni articoli viene impiegato il corsivo per dimostrare un distacco, evidenziare la natura metalinguistica di tale uso:

Se c'è un florilegio così folto sulla cretineria dei carabinieri, la ragione dev'essere cercata, dicono gli autori, nel comportamento vessatorio che i caramba hanno sempre avuto nei confronti dei deboli. (Francesco Rosso, Discorso serio sulla satira ridanciana, "La Stampa", 20/I/1978)

In un altro importante archivio storico, quello della "Repubblica", la prima attestazione si ritrova in un articolo dell'agosto del 1987 sulla fine della latitanza del famoso criminale Renato Vallanzasca, il quale, durante la cattura disse: "Bravi, caramba, avete fatto tredici!" (Roberto Bianchin, 'Bravo caramba, hai fatto tredici, "la Repubblica", 8/8/1987). L'archivio, però, raccoglie gli articoli soltanto a partire da 1984; è dunque altamente probabile che possano esserci attestazioni precedenti non documentate.

Una mera analisi quantitativa sul numero di occorrenze nei due repertori giornalistici ci mostra che caramba compare più o meno con la stessa frequenza tra gli anni Settanta e Novanta - per la precisione, 9 occorrenze negli anni Settanta, 6 negli anni Ottanta, 7 negli anni Novanta - per poi diffondersi notevolmente negli anni Duemila: sono i8 i casi riscontrati relativi a questo periodo, tenendo tra l'altro in considerazione che l'archivio della "Stampa" non va oltre il 20o6. L'elemento di novità risiede nell'uso di caramba con un intento maggiormente ludico: in un articolo sulle nuove uniformi delle carabiniere si citano le "caramba in gonnella" (Antonella Amapane, La carabiniera è chic e porta il tacco alto, "La Stampa", 23/I/200o); "più samba e meno caramba" recita una scritta su un muro del quartiere San Lorenzo, a Roma (Laura Laurenzi, Storie d'amore sul muro di casa, "la Repubblica", I8/I2/2012).

A proposito di finalità ludiche, abbiamo anche testimonianza dell'uso in LinguaGiovani, la banca dati online sul linguaggio giovanile realizzata dall'Università di Padova, che riporta caramba nella glossa di un'altra voce, cannare, mentre viene registrata come voce a sé stante la variante caraban, documentata alla Spezia ("i carabàn l'han fermato e gli han fatto una bella multa"); sempre in relazione ai repertori online, la banca dati itTenTen, uno dei più vasti corpora di italiano in rete, consultabile tramite il software di analisi testuale Sketch Engine, registra 592 occorrenze di caramba, a fronte però di oltre 4000 occorrenze di carabiniere e carabinieri.

È possibile ipotizzare, dunque, che caramba abbia cominciato stabilmente a circolare a partire dagli anni Sessanta e che sia ancora piuttosto diffuso nell'italiano contemporaneo, ma la scarsità di fonti disponibili non ci permette di andare oltre il dubbio: basti pensare al fatto che lo sterminato repertorio di Google libri registra un solo uso del vocabolo in un dialogo presente nel romanzo noir di Massimo Barone, Amici di chiave, edito a Roma da Fazi nel 1998 (a pagina 76): "I caramba lo cercano negli scafossi tra Tarquinia e Montericcio. Sanno che sta provando la Jeep", ha spiegato lo Svitato».

\section{(S)birro}

Tutt'altro che gergale è la parola sbirro, diffusa nella nostra lingua da secoli. L'etimologia del vocabolo non è certa: secondo il DELI, l'ipotesi più probabile è che derivi dal latino tardo birru(m), il mantello rosso a cappuccio che avrebbero indossato gli antichi sbirri, a sua volta proveniente dal greco pyrrós 'rosso'; a partire da birro, sbirro si sarebbe formato tramite l'aggiunta del prefisso latino ex-con valore peggiorativo (v. il GDLI). Il DELI avanza anche un'altra ipotesi, cioè che possa trattarsi di una 
variante di sgherro, parola che già anticamente designava l'uomo al servizio di un potente, dai modi violenti e intimidatori, alla stregua dei bravi e dei sicari (v. ancora il GDLI).

Il birro era nel Medioevo la guardia al servizio di una certa autorità civile, e fin da allora il vocabolo poteva avere una connotazione fortemente negativa, come si nota da uno dei due esempi che il TLIO riporta per il lemma, un brano tratto dall'anonima Lauda di San Torpé, composta agli inizi del XIV secolo:

Un di que' biri di mala ragione / trase la spada e taglioli la testa: / l'anima co gran festa / in ciel n’andò al suo sposo bëato. / Un di que' biri, per più diligione, / disse al co[m]pagnio: "Questo rinegato / avea grande piacere e devosione / che questo corpo fusse soterato...".

La prima impressione del Vocabolario della Crusca rimanda da birro alla voce famiglia, che poteva avere nell'italiano di allora il significato di "sergenti, o serventi della Corte"; a partire dalla seconda impressione birro viene assimilato a berroviere - più avanti anche birroviere, proprio per l'analogia con birro - che era al tempo "huomo di mal'affare", oppure stava a significare il donzello o il tavolaccino, entrambi servitori dei magistrati. In particolare, il tavolaccino era, in età comunale, il messo che durante le cerimonie pubbliche portava il "tavolaccio" con le insegne del comune (GDLI). Gli Accademici, inoltre, rimandano per birro alla voce latina littor, il littore che, nell'antica Roma, assisteva munito di fascio littorio le alte cariche pubbliche.

Birro e sbirro sono per lungo tempo due possibili varianti: se berroviere / birroviere sono registrati come arcaismi nel Tommaseo-Bellini, nel dare una definizione di birro Tommaseo avverte che in Toscana è "voce non men viva di Sbirro", anche se quest'ultima era più comune nel resto d'Italia, più familiare e maggiormente dispregiativa. Il GDLI inoltre riporta esempi di birro ancora a inizio Novecento, nell'opera del Cicognani (Sei storielle di nuovo conio, prima ed. I9I7-I8): "Torna a casa: se no, la strada, se ti vede abbandonato, t'inghiotte; o muori di fame se prima i birri non t'ànno ricondotto a forza dai tuoi o portato alle Stinche" (cioè alle carceri di Firenze).

Un ulteriore appunto sulla fortuna della parola: birro è l'appellativo che viene usato per definire il primo investigatore seriale nella storia del nostro poliziesco, il commissario Lucertolo, protagonista del ciclo di quattro romanzi di Giulio Piccini - in arte Jarro - edito da Treves (L'assassinio nel Vicolo della Luna e Il processo Bartelloni, pubblicati nel I883, I ladri di cadaveri e La figlia dell'aria, usciti l'anno seguente); per di più, nei romanzi i birri usano un proprio gergo, imparentato in parte con il furbesco, il gergo della malavita:

Un dialogo meno concitato seguiva tra i due birri di guardia alla porta.

- Ehi, Mengo! - diceva l'uno.

- Ehi, Mordente! - replicava l'altro.

[In nota: "Mengo, Mordente: nomi generali che i birri si davano fra loro nel gergo, che avevano, e al quale appartengono tutte le altre parole in corsivo, riferite nel dialogo".] (Jarro, L'assassinio nel Vicolo della Luna, Milano, Treves, 199I, edizione digitale a cura di Liber Liber)

Sempre nel Grande dizionario della lingua italiana, accanto a birro sono registrati gli alterati spregiativi birraccio, birracchiolo e birracchiuolo, birrucolo, cosi come molti sono i derivati altrettanto spregiativi di sbirro: un gruppo di sbirri viene definito sbirraglia o sbirreria; sbirrescamente è ciò che si fa con metodi repressivi, come sbirresco è ciò che è proprio degli sbirri, soprattutto con riferimento ai comportamenti "arroganti, prepotenti"; infine sbirrismo è sinonimo di arroganza e brutalità. A proposito di sbirro, il vocabolo è attestato a partire dal primo Cinquecento nelle opere di Sanudo (GDLI) e di Firenzuola (DELI). Il Vocabolario della Crusca registra la voce nella terza e nella quarta 
impressione, e in entrambi i casi la definizione che viene data è 'birro', così come nel TommaseoBellini. Secondo i dizionari storici non vi sono delle differenze sostanziali tra le due forme. Tuttavia solo una di esse, sbirro, è sopravvissuta nella lingua contemporanea.

Infatti, come rileva il GDLI, sbirro, nellitaliano novecentesco, ha assunto una connotazione maggiormente spregiativa "soprattutto in riferimento ai corpi di polizia di Stati e governi invisi per il carattere autoritario" e in questo senso la parola è ancora abbondantemente diffusa: nell'archivio della "Stampa" sono oltre 500o le occorrenze tra il 1970 e il 2006 - distribuite principalmente negli intervalli I970-1980 e I990-2006; nel repertorio della "Repubblica", relativamente al periodo I984-202I, le attestazioni sono più di i7oo. Sbirro e sbirri compaiono inoltre nei titoli di alcuni film italiani, per esempio Sbirro, la tua legge è lenta... la mia... no! poliziesco del 1979 diretto da Stelvio Massi, o Sbirri, documentario del 2009 sotto la direzione di Roberto Burchielli; allo stesso modo, il vocabolo appare nei titoli di numerosi romanzi gialli, come Il giovane sbirro di Gianni Biondillo (Parma, Guanda, 2007). Sono soltanto alcuni esempi che pero testimoniano un largo uso del vocabolo nell'italiano odierno, all'interno dei più diversi contesti.

\section{Cita come:}

Manuel Favaro, Origine e diffusione dicaramba e disbirro, "Italiano digitale", XVIII, 2021/3 (lugliosettembre)

DOI: $10.35948 / 2532-9006 / 2021.10604$

Copyright 2021 Accademia della Crusca

Pubblicato con licenza creative commons CC BY-NC-ND 